\title{
CONTRIBUTING FACTORS OF NON-ADHERENCE TO TREATMENT AMONG THE PATIENTS WITH TYPE II DIABETES MELLITUS
}

Bhattarai $\mathrm{B}^{1}$, Bista $\mathrm{B}^{2 \dagger}$, Shrestha $\mathrm{S}^{3}$, Budhathoki $\mathrm{B}^{4}$, Dhamala $\mathrm{B}^{5}$

${ }^{12345}$ Manmohan Memorial Institute of health Sciences, Soalteemode, Kathmandu

${ }^{\dagger}$ Corresponding author: Professor Beenu Bista, Department of Nursing MMIHS Nepal; Email: bistabeenu1@gmail.com

\section{ABSTRACT}

Background: The Multiple therapeutic component and need for life long treatment makes diabetic adherence a difficult task. We took this study to identify the Non adherence Patient to the Diabetic treatment, and to assess the contributing factors for non adherence.

Methods: A four questions preformed questionnaire -the Morisky medication adherence scale (MMAS) and Structured questionnaire were used to assess the contributing factors of Non adherence to diabetic treatment in 214 patient with type 2 diabetes mellitus which was carried out on Kathmandu Diabetic and Thyroid Center, Alka Hospital and Endocrine Care Center, Kupandol following Probability simple random sampling technique.

Results : A total of 55.14\% of diabetic client were non adherent .Major contributing factor of nonadherence to diabetic treatment were ignorance for life style modification i.e. 83.78\%, Among them, 59.48\% didn't take the prescribed medicine in time, most of them $85.71 \%$ didn't follow diabetic diet and less than half (46.61\% ) didn't monitored blood glucose level regularly due to poor self discipline. Gender, occupation and educational status were the significant contributors to Non adherence.

Conclusion : The findings showed high percentage of non adherent diabetic client and enlighten the various factors causing non adherence. It focuses the need for one to one counselling and constant motivation frequently to ensure better adherence.

Keywords: Diabetes, Non adherence, Morisky medication adherence scale 
INTRODUCTION

Globally according to WHO the number of people with diabetes has risen from 108 million in 1980 to 422 million in 2014. The global prevalence of diabetes among adults over 18 years of age has risen from 4.7\% in 1980 to 8.5\% in 2014 (WHO, 2016).According to WHO, the percentage of diabetic patients has increased from 19.04\% in 2002 to $25.9 \%$ in 2009 in Nepal. ${ }^{1}$

Non-adherence to therapeutic strategies is a serious concern that poses a great challenge to the successful delivery of healthcare. Patient non-adherence is not only limited to the failure to take medication but also the failure to make lifestyle changes, undergo tests or keep appointments with physicians. The non-adherent patients especially with chronic disease are more prone to encountering serious difficulties. ${ }^{2}$

Non-adherence can be due to factors that are patient centered, therapy related or health care system related. The patient centered factors can be demographic (age, gender, educational level and marital status) and psychological (patients beliefs and motivation towards the therapy, negative attitude, patient-prescriber relationship, understanding of health issues and patient knowledge). The therapy related factors include route of medication, duration of treatment, complexity of treatment and side effects of the medicines. The factors associated with the health care system include availability, accessibility and the physician. ${ }^{2}$

The prevalence of therapeutic non-adherence in 150 known Type 2 DM patients, 82 patients (54.66\%) were non adherent. Among the non-adherent patients, 16 patients (17.08\%) were illiterates, 32 patients (39.02\%) had economic problems to buy prescribed medications, 53 (64.63\%) of them had lack of information about prescribed medications was seen, 37 (45.12\%) patients were not aware of the side effects of the prescribed medication, 59 (71.95\%) patients were not aware of what happens on missing medications regularly, 39 (47.56\%) of them were not able to visit physician regularly for consultation, 52 (63.45\%) and 49 (59.75\%) of patients were not performing exercise and not following advise on diet respectively. ${ }^{11}$

The assessment of the patient's responses to the 4-item modified Morisky adherence predictor scale showed that out of 105 DM Patients 43 (40.95\%) of the patients had good adherence with 
prescribed medications, whereas 39 (37.14\% ) had medium adherence and 23 (21.90 \%) had low adherence. $^{3}$

Prevalence of DM is growing rapidly worldwide. And most of the diabetic patients do not follow the treatment regimen properly due to different reasons. ${ }^{4-6}$

Most of the previous studies were done in developed countries, so in Nepal, limited study was done related to prevalence of non-adherence and contributing factors. ${ }^{7}$ Therefore this study was carried out to assess the prevalence of non adherence and identify the factors contributing to non adherence to diabetic treatment.

\section{MATERIAL AND METHODS}

A cross sectional study design was conducted in diabetic clinic of Kathmandu Diabetic and Thyroid Center, Private Limited, Alka Hospital and Endocrine Care Center, Kupandol. during the period of 8 weeks. Simple random sampling was carried out for the selection of 214 patient with Type II DM aged 20 years and above receiving diabetic treatment since 1 year attending at diabetic clinic. An ethical approvals obtained from institutional review committee (IRC)MMIHS.

The voluntary consent was taken after expelling the purpose of the study to the individual patient.

The selected patient were interviewed through the structured questionnaire regarding sociodemographic characteristics and reasons for non adherence to diabetic treatment which includes pharmacological and non pharmacological treatment (Oral hypoglycaemic drugs with diet, exercise and followup).

Non-adherence was assessed through the specific four item Morisky Medication Adherence Scale (MMAS-4) has high reliability and validity,and the patient is considered non adherent if he /she answered yes to one out of four ,Morisky’s Medication Adherence scale (MMAS) Type II DM.,9 The different variables were presented in frequency and percentage . The chi -square test was used to show the significance of association between group (p-value of 0.05 or less was considered as statistically significant . 


\section{RESULTS}

A total 214 patient attended the Diabetic clinic during 8 weeks period who met the defined inclusion criteria.

More than half $69.16 \%$ of respondents were from the age group $40-59$ years. Nearly half (51.4\%) of the respondents were female. A total of 200 (93.46\%) of the patients included in the study were married. Most (66.82\%) of the respondents were literate and out of eighty one (37.85\% ) studied from secondary up to higher secondary level .Eighty five (39.72\%) were doing their own business. Majority of the respondents $60.75 \%$ received anti-diabetic treatment for 3 to 5 years. The percentage of Non Adherent respondents to diabetic treatment was presented in Table 1.

Table 1. Prevalence of Adherent and Non Adherent to Diabetic Treatment

\begin{tabular}{lcc}
\hline Diabetic treatment & Frequency & $\mathbf{\%}$ \\
\hline Adherent & 96 & 44.86 \\
Non adherent & 118 & 55.14
\end{tabular}

Of total, more than a half 55.14\% of patients self-reported non adherence to their anti diabetic drug regimens. according to Morisky's instrument. The contributing factors for non adherent were tabulated on Table 2.

Table 2. Contributing Factors for Non Adherence to Diabetic Treatment

$\mathrm{n}=\mathbf{1 1 8}$

\begin{tabular}{lcc}
\hline Contributing factors & Frequency & $\mathbf{\%}$ \\
\hline Complexity of medication. & 22 & 19.82
\end{tabular}


Unwillingness to take prescribed medication.

Lack of information about prescribed medication.

Unaware about missed drug.

Ignorance for life style modification.
35

31.53

8

61

54.95

93

The contributing factors for non-adherence to diabetic treatment were ignorance to life style modification (83.78\%), unaware about miss drugs (54.95\%), unwillingness to take prescribed medicine (31.53\%), complexity of medication (19.82\%), cost of prescribed anti-diabetic drugs (15.32\%) and lack of information about prescribed medicine (7.21\%).

Though most of the patient got information from Pharmacist, 59.58\% didn't take their prescribed medicine in times due to poor discipline.

Almost all patient got information (about exercise,Diabetic diet,Follow up) but 63.56\% didn't perform exercise regularly due to extreme weather condition ,85.71\% not followed diabetic diet due to poor self discipline and a half of the patient didn't go for follow up regularly due to far distance from home and busy schedule .

Despite knowing the normal blood glucose level (78.81\%), less than half $46.61 \%$ didn't monitor their blood glucose level regularly due to poor self discipline. Association between respondent's Non Adherence to diabetic treatment due to cost of prescribed medication with socio demographic characteristics are presented in Table 3. 
Table 3. Association between Non Adherence to Diabetic Treatment due to cost of Prescribed Medication and Socio-Demographic Characteristics

$$
\mathrm{n}=\mathbf{1 1 8}
$$

\begin{tabular}{|c|c|c|c|}
\hline \multirow[t]{3}{*}{ Variables } & \multicolumn{2}{|c|}{ Non-adherence to Diabetic Treatment } & \multirow[t]{3}{*}{ p-value } \\
\hline & Yes & No & \\
\hline & n (\%) & n (\%) & \\
\hline
\end{tabular}

\section{Age}

20-59 years

13 (11.07)

71 (60.17)

0.603

60 and above

$4(3.39)$

$30(25.42)$

Gender

Male

$3(2.54)$

$56(47.46)$

0.004

Female

14 (11.86)

45 (38.14)

Marital status

Single

$0(0)$

$1(0.85)$

0.576

Married

100 (84.75)

Educational status

Literate

72 (61.02)

0.015

Illiterate

10 (8.47)

29 (24.58)

Occupation

Employed

4 (3.39)

66 (55.93)

0.003

Unemployed

13 (11.02)

35 (29.66)

Non adherence to Diabetic Treatment to cost of prescribed medication was associated with occupation, gender and education. Association between Respondent's Non Adherence to diabetic treatment due to unaware about missed drugs with their socio demographic characteristics are presented in Table 4 
Table 4. Association between Non Adherence to Diabetic Treatment due to Unaware about Missed Drug and Socio-Demographic Characteristics

$$
\mathbf{n}=118
$$

Variables Non-adherence to Diabetic Treatment $\quad$ p-value

$\begin{array}{cc}\text { Yes } & \text { No } \\ \text { n (\%) } & \text { n (\%) }\end{array}$

\section{Age}

20-59 years

45 (38.14)

39 (33.05)

0.521

60 and above

16 (13.56)

18 (15.25)

Gender

Male

28 (23.73)

31 (26.27)

0.357

Female

33 (27.97)

$26(22.03$

Marital status

Single

$1(0.85)$

0 (0)

0.249

Married

60 (50.85)

57 (48.31)

Educational status

Literate

32 (27.12)

47 (39.83)

0.001

Illiterate

29 (24.58)

10 (8.47)

Occupation

Employed

32 (27.12)

38 (32.2)

0.116

Unemployed

29 (24.58)

19 (16.1)

Educational status was found significantly associated with Non Adherence to diabetic treatment due to unaware about missed drug. Association between Respondent's Non Adherence to diabetic 
treatment due ignorance for lifestyle modification with their socio demographic characteristics are presented in Table 5

Table 5. Association between Non Adherence to Diabetic Treatment due to ignorance for Life Style Modification and Socio-Demographic Characteristics

$\mathbf{n}=\mathbf{1 1 8}$

\begin{tabular}{|c|c|c|c|}
\hline \multirow[t]{2}{*}{ Variables } & \multicolumn{2}{|c|}{ Non-adherence to Diabetic Treatment } & \multirow[t]{2}{*}{ p-value } \\
\hline & $\begin{array}{c}\text { Yes } \\
\text { n (\%) }\end{array}$ & $\begin{array}{c}\text { No } \\
\text { n (\%) }\end{array}$ & \\
\hline \multicolumn{4}{|l|}{ Age } \\
\hline 20-59 years & 64 (54.24) & 20 (16.95) & 0.273 \\
\hline 60 and above & $29(24.58)$ & $5(4.24)$ & \\
\hline \multicolumn{4}{|l|}{ Gender } \\
\hline Male & $37(31.36)$ & $22(18.64)$ & 0.001 \\
\hline Female & $56(47.46)$ & $3(2.54)$ & \\
\hline \multicolumn{4}{|l|}{ Marital status } \\
\hline Single & $1(0.85)$ & $0(0)$ & 0.489 \\
\hline Married & $92(77.97)$ & 25 (21.19) & \\
\hline \multicolumn{4}{|c|}{ Educational status } \\
\hline Literate & $60(50.85)$ & $19(16.1)$ & 0.279 \\
\hline Illiterate & 33 (27.97) & $6(5.08)$ & \\
\hline \multicolumn{4}{|l|}{ Occupation } \\
\hline Employed & $48(40.68)$ & $22(18.64)$ & 0.002 \\
\hline Unemployed & 45 (38.14) & $3(2.54)$ & \\
\hline
\end{tabular}


Highly significant association was found between gender, occupation and ignorance for life style modification.

\section{DISCUSSION}

More than half respondents 55.14\% were non adherent to their diabetic treatment. In comparison to these finding two studies conducted in India showed that the patients self-reported non adherence rate to anti-diabetic treatment was $83.33 \%$ and $54.66 \% .{ }^{9,11}$ which was comparatively higher and similar to this study.

The most common reasons for non adherence to prescribed medications were modifiable factors that could be overcome by adopting suitable measures. Poor self discipline (49.48\%) was the most commonly mentioned reason for non adherence which is different from study conducted in Tanzania where the main reasons for not taking prescribed medication in time due to high cost of anti-diabetic drugs (52.7\%), drug side effects $(11.49 \%)^{10}$

The contributing factors for non adherence to diabetic treatment in this study were due to ignorance for life style modification (83.78\%), unaware about missed drug (54.95\%), unwillingness to take prescribed medication (31.53\%), complexity of medication (19.82\%), due to cost of prescribed anti-diabetic drug (15.32\% ) and lack of information about prescribed medication (7.21\%) is different from study conducted in India where (39.02\%) had economic problems to buy prescribed medications, (64.63\%) had lack of information about prescribed medications, (45.12\%) patients were unaware of the side effects of the prescribed medication, (71.95\%) patients were unaware of what happens on missing medications regularly. ${ }^{11}$

Present study noted significant association between occupation, gender and educational status with Non adherence to diabetic treatment with cost of prescribed medication. Similarly association was found between educational status with Non adherence to diabetic treatment with unaware about missed drug. And also there was highly significant association between gender and occupation with Non adherence to diabetic treatment (ignorance for life style modification). This study was supported by other studies where factors found to be significantly associated with non-adherence on bivariate analysis were: sex $(<0.001)$, education level $(0.02){ }^{5}$ 


\section{CONCLUSION}

The study reveals that the high number of diabetic patient were non adherent to diabetic treatment. The findings showed that the major contributing factors for non-adherence to diabetic treatment were due to ignorance for life style modification and unaware about missed drug and very few of them due to lack of information about prescribed medication. The association between selected variables and non-adherence to diabetic treatment was highly significant (gender, occupation, cost of prescribed medication, education status, and unaware about missed drug).

Therefore, there is a definite need to improve patient adherence by hospital and community based awareness program, targeted on importance of life style modification, continue motivation and continuity of diabetic drug to patient and their families.

\section{REFERENCES}

Yadav. Assessment of Diabetes Mellitus in India and Nepal. 2012. Available from: https://www.webmedcentral.com/article_view/3544

Khan, Lateef, Aithan \& Khamseen. Factors Contributing to Non-Compliance among Diabetes Attending Primary Health Centers in the AI Hasa District of Saudi Arabia. Department of Community Ophthalmology, AI Omran Primary Health Care Center. 2012: 1-7.

Sajith, Pankaj, Pawar, Modi \& Sumariya. Medication Adherence to Antidiabetic Therapy in Patients eith Type 2 Diabetes Mellitus. Department of Clinical Pharmacy. 2013: 1-7.

Ganlyu, Mabuza, Malate, Govender \& Ogunbanjo. Non-adherence to diet and exercise recommendations amongst patients with type 2 diabetes mellitus attending Extension II Clinic in Botswana. Department of Family Medicine and Primary Health Care, University of Limpopo, South Africa. 2013: 1-6.

Kalyango, Owino \& Nambuya. Non-adherence to diabetes treatment at Mulago Hospital in Uganda:prevalence and associated factors. Department of Pharmacy. 2008: 1-7. 
Sharma, S K. Nursing Research and Statistics New Delhi: Reed Elsevier India Private Limited: 2014.

Gelow, B K. et al. Nonadherence and Contributing Factors among Ambulatory Patients with Antidiabetic Medications in Adama Referral Hospital. Department of Pharmacy. 2014: 1-7.

Morisky,DE,Green ,LW,Levine DM.Concurrent \& predictive validity of a self reported measures of medication adherence. Med Care 1986;24:67-74

Sharma, Kalra ,Dhasmana \& Basera .Poor adherence to treatment : A major challenge in diabetes .Indian Academy of clinical medicine. 2014 :1-4

Rwegerera. Adherence to anti-diabetic drugs among patients with Type 2 diabetes mellitus at Muhimbili National Hospital, Dar es Salaam, Tanzania. Pan African Medical Journal: 2014: 1-9.

Divya, Nadig. Factors Contributing to Non-Adherence to Medication among Type 2 Diabetes Mellitus in Patients Attending Tertiary Care Hospital in South India. 2015:274-276.

Lavsa S. M., Holzworth A., Ansani N. T. Selection of a validated scale for measuring medication adherence. Journal of the American Pharmacists Association. 2011;51(1):90-94. doi: 10.1331/JAPhA.2011.09154.

Tan X., Patel I., Chang J. Review of the four item Morisky Medication Adherence Scale (MMAS-4) and eight item Morisky Medication Adherence Scale (MMAS-8) Innovations in Pharmacy. 2014;5(3):p. 165. 